\title{
On Minimal Words With Given Subword Complexity
}

\author{
Ming-wei Wang* \\ Department of Computer Science \\ University of Waterloo \\ Waterloo, Ontario N2L 3G1 \\ CANADA \\ m2wang@neumann.uwaterloo.ca
}

\author{
Jeffrey Shallit* \\ Department of Computer Science \\ University of Waterloo \\ Waterloo, Ontario N2L 3G1 \\ CANADA \\ shallit@graceland.uwaterloo.ca
}

Submitted: May 28, 1998; Accepted: July 15, 1998.

\begin{abstract}
We prove that the minimal length of a word $S_{n}$ having the property that it contains exactly $F_{m+2}$ distinct subwords of length $m$ for $1 \leq m \leq n$ is $F_{n}+F_{n+2}$. Here $F_{n}$ is the $n$th Fibonacci number defined by $F_{1}=F_{2}=1$ and $F_{n}=F_{n-1}+F_{n-2}$ for $n>2$. We also give an algorithm that generates a minimal word $S_{n}$ for each $n \geq 1$.
\end{abstract}

1991 Mathematics Subject Classification: Primary 68R15; Secondary 05C35.

\section{Introduction}

In this paper we solve a particularly interesting case of the following more general problem. Let $f: \mathbb{N} \longrightarrow \mathbb{N}$ be a non-decreasing function. Given a word $w$, a subword of $w$ is any contiguous block of symbols of $w$. For each word $w$ over some fixed finite alphabet, we define $P_{w}(n)$ to be the number of distinct subwords of $w$ of length $n$. We say that $f$ is feasible if for each integer $N \geq 1$ there exists at least one word $w=w(N)$ such that $P_{w}(n)=f(n)$ for $1 \leq n \leq N$. Such words $w(N)$ are said to possess the property $\mathcal{P}_{f(N)}$. At the present, there is no known simple characterization of the class of feasible functions. If $f$ is feasible, let us call a shortest word $w$ possessing property $\mathcal{P}_{f(N)}$ a minimal word of order $N$ with respect to $f$. Then several natural questions can be asked.

\footnotetext{
${ }^{*}$ Supported in part by a grant from NSERC Canada.
} 
1. What is the length of a minimal word of order $N$ ?

2. Is there a reasonably efficient algorithm that finds such minimal words?

3. For each order how many minimal words are there?

We show that the function $f(n)=F_{n+2}$ is feasible, give an algorithm that finds a minimal word of order $n$ for each $n$ and show that the length of a minimal word of order $n$ is $F_{n}+F_{n+2}$ for $n>1$. However, the question of a complete enumeration of all minimal words of order $n$ is still open. Here the $F_{i}$ are the Fibonacci numbers defined by $F_{1}=F_{2}=1$ and $F_{n}=F_{n-1}+F_{n-2}$ for $n>2$. Previously Good [G46] showed that the length of a shortest word containing as subwords all $2^{n}$ binary words of length $n$ is $2^{n}+n-1$. In the same year de Bruijn [B46] gave a complete enumeration of all such words (see also [B75]).

The converse problem is usually formulated as finding the function $f$ when given a set of words $w$. When the words $w$ are the prefixes of some infinite sequence $S$, the function $f$ is one measure of the complexity of $S$, and is usually referred to as the subword complexity of $S$. For related results on subword complexity see the survey article of Allouche [A94].

The proof of our results centers on a detailed analysis of a version of the de Bruijn graph which appeared first implicitly in [F94] and explicitly in [R83]. Good [G46] and de Bruijn [B46] independently defined a version of these graphs in 1946. See Fredricksen [F82] for more references for the de Bruijn graph. Observe that $f(1)=F_{3}=2$, which means the number of distinct subwords of length 1 is 2 . Thus we need only consider binary words over $\{0,1\}$ in the rest of this paper.

We divide the presentation of the proof into 4 parts:

1. Existence

2. Structure of the word graph

3. Lower bound on the length

4. Algorithm that generates words which achieve the lower bound

\section{Existence}

In this section we establish the existence of words with property $\mathcal{P}_{f(n)}$ for each $n$. The method we employ leads to the de Bruijn graphs. We will define these graphs in this section and use them to prove our result in subsequent sections.

Lemma 1.1 Let $S_{n}$ denote the set of words of length $n$ that omit 11 . Then $\left|S_{n}\right|=F_{n+2}$ for all integers $n \geq 1$.

Proof: We proceed by induction. The case $n=1$ and $n=2$ are trivial. For the inductive step note that $S_{n}$ can be partitioned into two sets $S_{n, 0}$ and $S_{n, 1}$ where $S_{n, 0}$ contains words that begin with 0 and $S_{n, 1}$ contains words that begin with 1 . Since no word of $S_{n}$ contains 11, it is easy to see that $\left|S_{n, 1}\right|=\left|S_{n-2}\right|$ and $\left|S_{n, 0}\right|=\left|S_{n-1}\right|$. Thus we have $\left|S_{n}\right|=\left|S_{n-1}\right|+\left|S_{n-2}\right|$. 
The Fibonacci numbers satisfy the same recurrence relation. Since we verified the initial condition $S_{1}=F_{3}$ and $S_{2}=F_{4}$, the lemma is proved.

Remark: Let $w$ be a word of $S_{i}$. Then $w 0^{j-i}$ is a member of $S_{j}$ if $j \geq i$. Hence every word of $S_{i}$ occurs as a subword of some word of $S_{j}$ if $i \leq j$.

Theorem 1.1 There exist finite words with property $\mathcal{P}_{f(n)}$ for each $n>0$.

Proof: Let $S_{n}=\left\{w_{1}, w_{2}, \ldots, w_{m}\right\}$. Then the word $w_{1} 0 w_{2} 0 \ldots w_{n}$ possesses property $\mathcal{P}_{f(n)}$ by Lemma 1.1 and the remark above.

Note that Theorem 1.1 gives an upper bound of $n F_{n+2}+n-1$ for the length of a minimal word of order $n$. The next theorem shows that the above construction is essentially unique.

Theorem 1.2 For all $n>2$, any finite word $w$ possessing property $\mathcal{P}_{f(n)}$ omits either 00 or 11.

Proof: Since $n>2, P_{w}(2)=3$, and so $w$ omits either 00, 01, 10, or 11 . If it omits 01 then $w \in 1^{*} 0^{*}$ and hence all subwords of $w$ of length 3 are contained in $\{111,110,100,000\}$. This implies $P_{w}(3) \leq 4$. However $P_{w}(3)=F_{5}=5$, a contradiction. A similar argument shows $w$ cannot omit 10. Therefore $w$ omits either 00 or 11 .

\subsection{Word graph}

We define the particular kind of de Bruijn graphs that we use below. An example is shown in Figure 1.

Definition 1.1 For $n>0$, the word graph $G_{n}$ is a directed graph with labeled edges defined as follows.

- The vertices of $G_{n}$ are all words of length $n$ that omit 11.

- The edges of $G_{n}$ consist of all pairs of vertices $(a w, w b)$ with label $b$ such that aw $\neq w b$ and $a, b \in\{0,1\}$. 


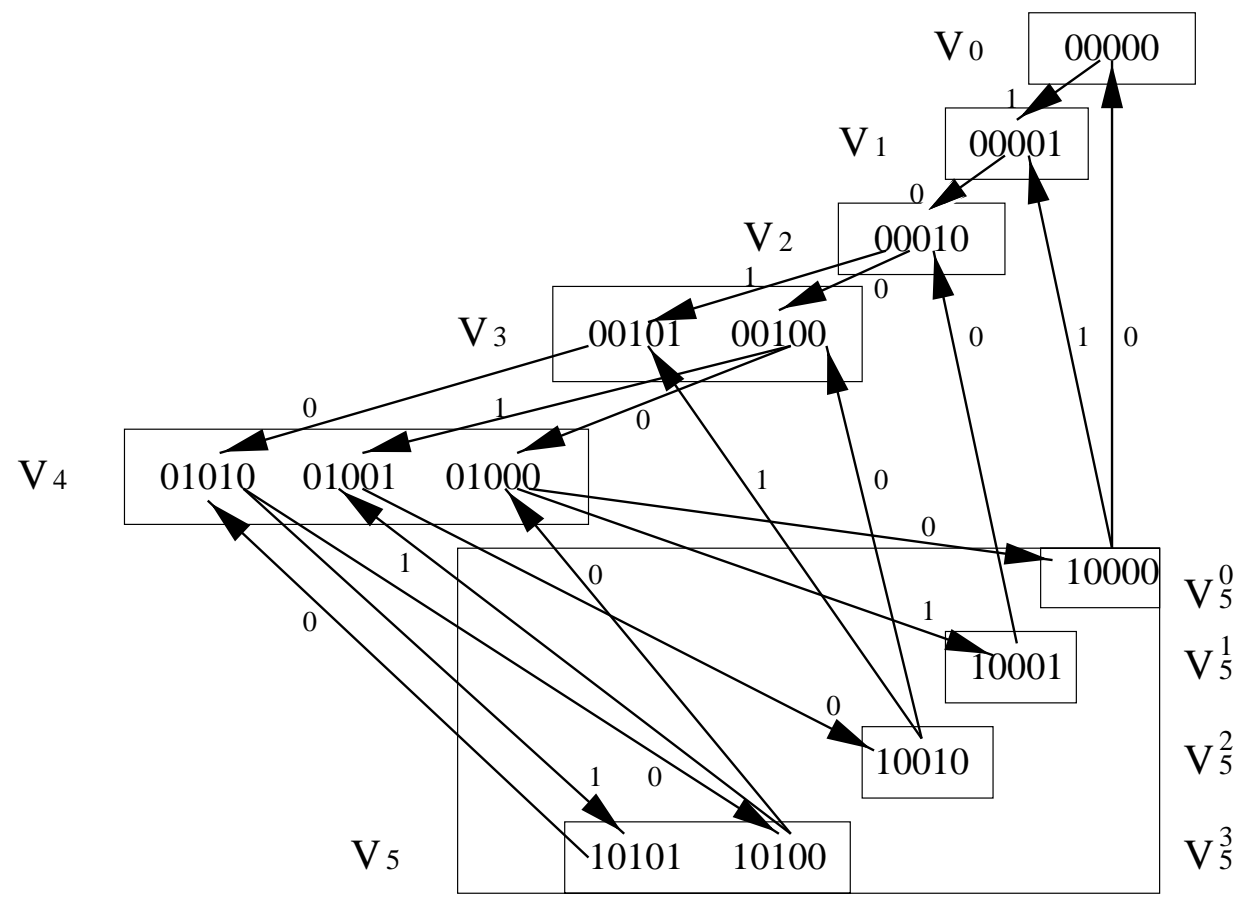

Figure 1: The directed graph $G_{5}$.

Let $L(n)$ be the minimal length of a word $w$ possessing property $\mathcal{P}_{f(n)}$. A walk in a graph $G$ is a sequence of vertices $\left\{P_{1}, P_{2}, \ldots, P_{m}\right\}$ of $G$ such that $\left(P_{i}, P_{i+1}\right)$ is an edge in $G$ for $1 \leq i \leq m-1$. Note that a walk may repeat both vertices and edges. Let $l(n)$ be the length (number of edges traversed) of a shortest walk through $G_{n}$ which visits every vertex of $G_{n}$ at least once. Then Theorem 1.1 and Theorem 1.2 together imply that for $n>2$, $L(n)=l(n)+n$. In subsequent sections we prove that $l(n)=F_{n}+F_{n+2}-n$.

\section{Structure of the word graph}

We summarize few properties of $G_{n}$ in the following lemma. These properties can be seen more easily by contemplating Figure 1 . We say that a graph $G$ is $n$-partite if the vertices of $G$ can be partitioned into $n$ sets such that there are no edges between any pair of vertices in the same partition.

Lemma 2.1 Let $G_{n}=G=(V, E)$ be a word graph, and $n>2$. Then $G$ has the following properties.

1. $V$ can be partitioned into disjoint subsets $V_{0}, V_{1}, \ldots, V_{n}$ where $V_{i}$ consists of words that begin with exactly $(n-i)$ 0's. In addition, $V_{n}$ can be partitioned into $n-1$ disjoint subsets $V_{n}^{0}, \ldots, V_{n}^{n-2}$ where each $V_{n}^{i}$ consists of words of $V_{i}$ with the first character changed to 1 .

2. We have $\left|V_{0}\right|=1,\left|V_{i}\right|=F_{i}$ for $1 \leq i \leq n,\left|V_{n}^{0}\right|=1$ and $\left|V_{n}^{i}\right|=F_{i}$ for $1 \leq i \leq n-2$. 
3. $G$ is an $(n+1)$-partite graph with the $V_{i}$ 's as partitions.

4. For $1 \leq i \leq n-1$, each vertex in $V_{i}$ has in-degree 2 and out-degree 1 or 2 ; each vertex in $V_{n}$ has in-degree 1 and out-degree 1 or 2.

5. Vertices in $V_{i}$ point only to vertices in $V_{i+1}$ for $0 \leq i \leq n-1$; vertices in $V_{n}^{i}$ point only to vertices in $V_{i+1}$ for $0 \leq i \leq n-2$ with the exception that $V_{n}^{0}$ also points to $V_{0}$.

These properties of $G_{n}$ are immediate from the definition. We omit the proof here.

\section{Lower Bound}

In this section we prove that $l(n) \geq F_{n}+F_{n+2}-n$ for $n>1$. Due to certain boundary conditions, results in this section are proved for $n>2$. The case $n=2$ can be proved by inspecting $G_{2}$ in Figure 2.

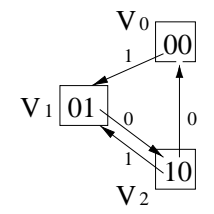

Figure 2: The directed graph $G_{2}$.

The following lemma is an easy consequence of parts (1) and (2) of Lemma 2.1.

Lemma $3.1 F_{n+2}=1+\sum_{i=1}^{n} F_{i}$ for $n \geq 1$.

Now let $G=G_{n}$ be a word graph. By a complete walk of $G$ we mean a walk through $G$ that visits each vertex of $G$ at least once. We begin by proving a lower bound on the length of a special type of complete walk of $G_{n}$. Then we will sketch the proof that the lower bound thus obtained is a lower bound for all complete walks of $G_{n}$.

Lemma 3.2 For $n>2$, if a complete walk of $G=G_{n}$ starts in $V_{n}$ and ends in $W=$ $V_{n}^{0} \cup V_{n}^{1} \cup V_{0} \cup V_{1}$, then it has length $\geq F_{n+2}+F_{n}-n$.

Proof: Define $V_{i}$ and $V_{n}^{i}$ as in Lemma 2.1. Fix an arbitrary complete walk $P$ in $G$ with the appropriate start and end points. Let $y_{i}$ be the total number of visits by $P$ to vertices of $V_{i}$ for $0 \leq i \leq n$. Let $x_{i}$ be the number of visits to vertices of $V_{n}^{i}$ for $0 \leq i \leq n-2$.

Since $P$ starts in $V_{n}$ and ends in $W$, it follows that all visits to $V_{i+1}(2 \leq i \leq n-2)$ must be preceded by a visit to either $V_{i}$ or $V_{n}^{i}$, and all visits to $V_{i}$ and $V_{n}^{i}$ are followed by a visit to $V_{i+1}$. Hence we see that $y_{i}+x_{i}=y_{i+1}$ or equivalently $y_{i}=y_{i+1}-x_{i}$ for $2 \leq i \leq n-2$. Furthermore since $P$ starts in $V_{n}$, using part 5 of Lemma 2.1 we have $y_{n}=y_{n-1}+1$ or equivalently $y_{n-1}=y_{n}-1$. Since $y_{n}=\sum_{i=0}^{n-2} x_{i}$ by definition, we have $y_{n-1}=y_{n}-1=\left(\sum_{i=0}^{n-2} x_{i}\right)-1$. 
Now for $2 \leq j \leq n-2$, we claim that

$$
y_{j}=\left(\sum_{i=0}^{j-1} x_{i}\right)-1 \quad(2 \leq j \leq n-1)
$$

The above system of equations can be established by a "downward induction" as follows. First note that we already have $y_{n-1}=\left(\sum_{i=0}^{n-2} x_{i}\right)-1$, so inductively assume $y_{j}=\left(\sum_{i=0}^{j-1} x_{i}\right)-$ 1 for $3 \leq j \leq n-1$. Now since $y_{j-1}=y_{j}-x_{j-1}$ we have by the induction hypothesis,

$$
\begin{aligned}
y_{j-1} & =y_{j}-x_{j-1} \\
& =\left(\sum_{i=0}^{j-1} x_{i}\right)-1-x_{j-1} \\
& =\left(\sum_{i=0}^{j-2} x_{i}\right)-1
\end{aligned}
$$

Thus by induction, (1) is proved.

Now we estimate the value of $y_{j}$ for each $j$. Since $P$ is a complete walk, by part 2 of Lemma 2.1 we have $x_{0} \geq\left|V_{n}^{0}\right|=1$ and $x_{i} \geq\left|V_{n}^{i}\right|=F_{i}$ for $1 \leq i \leq n-2$. Therefore using the system of equations in (1) we obtain the following system of estimates for $y_{j}(2 \leq j \leq n-1)$.

$$
\begin{aligned}
y_{j} & =\left(\sum_{i=0}^{j-1} x_{j}\right)-1 \\
& \geq 1+\left(\sum_{i=1}^{j-1} F_{j}\right)-1 \quad(\text { By Lemma } 3.1) \quad(2 \leq j \leq n-1) \\
& =F_{j+1}-1 \quad(2 \leq n)
\end{aligned}
$$

Trivially we also have $y_{0} \geq\left|V_{0}\right|=1, y_{1} \geq\left|V_{1}\right|=1$ and $y_{n} \geq\left|V_{n}\right|=F_{n}$. Now the length of $P$ can be bounded from below by these estimates as follows. 


$$
\begin{aligned}
|P| & =\left(\sum_{i=0}^{n} y_{i}\right)-1 \\
& =y_{0}+y_{1}+\left(\sum_{j=2}^{n-1} y_{j}\right)+y_{n}-1 \\
& \geq 1+1+\left(\sum_{j=2}^{n-1}\left(F_{j+1}-1\right)\right)+F_{n}-1 \\
& =2+\left(F_{n+2}-3\right)-(n-2)+F_{n}-1 \\
& =F_{n}+F_{n+2}-n
\end{aligned}
$$

Since $P$ is arbitrary, we see that $F_{n}+F_{n+2}-n$ is a lower bound for this type of complete walk.

Now we sketch the proof that $F_{n}+F_{n+2}-n$ is a lower bound for all complete walks of $G_{n}$. Suppose $P$ is a complete walk of $G_{n}$ that either does not start in $V_{n}$ or does not end in $W$. We associate the numbers $a$ and $b$ with the start and end points of $P$ respectively as follows. The number $a$ is the index of the partition where $P$ starts, i.e. $P$ starts in $V_{a}$. The number $b$ is slightly more complicated. If $P$ ends in $V_{i}(0 \leq i \leq n-1)$, then $b=i$. Otherwise $P$ ends in $V_{n}^{i}(0 \leq i \leq n-2)$, and we let $b=i$. In other words, we do not worry about where $P$ starts in $V_{n}$ but we do worry about where $P$ ends in $V_{n}$. There are four cases.

1. $a=b+1$. Then we have $y_{i}+x_{i}=y_{i+1}$ for $2 \leq i \leq n-1$. Therefore the system of equations in (1) of Lemma 3.2 is in this case replaced by

$$
\begin{aligned}
y_{j} & =y_{j+1}-x_{j} \\
& =\sum_{i=0}^{j-1} x_{i} \quad(2 \leq j \leq n-1)
\end{aligned}
$$

By same method as in (2) and (3) of Lemma 3.2, we arrive at a lower bound of $F_{n}+F_{n+2}-2$.

2. $2 \leq a \leq b$. Then we have $y_{a-1}+x_{a-1}+1=y_{a}$ and $y_{b}+x_{b}=y_{b+1}+1$ and $y_{i}+x_{i}=y_{i+1}$ for $i \neq a-1$ or $b, 2 \leq i \leq n-1$. In this case (1) is replaced by

$$
\begin{aligned}
& y_{j}=y_{j+1}-x_{j} \quad=\sum_{\substack{i=0 \\
b-1}}^{j-1} x_{i} \quad b<j \leq n-1 . \\
& y_{b}=y_{b+1}-x_{b}+1=\left(\sum_{i=0} x_{i}\right)+1
\end{aligned}
$$




$$
\begin{aligned}
& y_{j}=y_{j+1}-x_{j} \quad=\left(\sum_{i=0}^{j-1} x_{i}\right)+1 \quad a \leq j \leq b-1 \\
& y_{a-1}=y_{a}-x_{a-1}-1=\sum_{i=0}^{a-2} x_{i} \\
& y_{j}=y_{j+1}-x_{j} \quad=\sum_{i=0}^{j-1} x_{i} \quad 2 \leq j \leq a-2
\end{aligned}
$$

and (2) is replaced by

$$
\begin{aligned}
& y_{j}=\sum_{i=0}^{j-1} x_{i} \quad \geq F_{j+1} \quad b<j \leq n-1 . \\
& y_{b}=\left(\sum_{i=0}^{b-1} x_{i}\right)+1 \geq F_{b+1}+1 \\
& y_{j}=\left(\sum_{i=0}^{j-1} x_{i}\right)+1 \geq F_{j+1}+1 \quad a \leq j \leq b-1 \\
& y_{a-1}=\sum_{i=0}^{a-2} x_{i} \quad \geq F_{a} \\
& y_{j}=\sum_{i=0}^{j-1} x_{i} \quad \geq F_{j+1} \quad 2 \leq j \leq a-2
\end{aligned}
$$

Finally in place of (3) we have

$$
\begin{aligned}
|P| & =\left(\sum_{i=0}^{n} y_{i}\right)-1 \\
& =y_{0}+y_{1}+\left(\sum_{j=2}^{a-1} y_{j}\right)+\left(\sum_{j=a}^{b} y_{j}\right)+\left(\sum_{j=b+1}^{n-1} y_{j}\right)+y_{n}-1 \\
& \geq 1+1+\left(\sum_{j=2}^{a-1} F_{j+1}\right)+\left(\sum_{j=a}^{b}\left(F_{j+1}+1\right)\right)+\left(\sum_{j=b+1}^{n-1} F_{j+1}\right)+F_{n}-1 \\
& =2+\left(\sum_{j=2}^{n-1} F_{j+1}\right)+(b-a+1)+F_{n}-1 \\
(\text { By Lemma 3.1) } & =2+\left(F_{n+2}-3\right)+(b-a+1)+F_{n}-1 \\
& =F_{n}+F_{n+2}+b-a-1
\end{aligned}
$$

Thus we obtain a lower bound of $F_{n}+F_{n+2}+b-a-1$.

3. $a>b+1$. If $b \geq 2$, then this case is similar to case 2 with the equation $y_{b}=y_{b+1}-x_{b}+1$ switching position with the equation $y_{a-1}=y_{a}-x_{a-1}-1$ in (5). The lower bound 
derived is again $F_{n}+F_{n+2}+b-a-1$. If $b=0$ or 1 , then we have $a \leq n-1$ and the equations in (5) become

$$
\begin{aligned}
y_{j}=y_{j+1}-x_{j} & =\sum_{\substack{i=0 \\
a-2}}^{j-1} x_{i} \quad a \leq j \leq n-1 . \\
y_{a-1}=y_{a}-x_{a-1}-1 & =\left(\sum_{\substack{i=0 \\
j-1}} x_{i}\right)-1 \\
y_{j}=y_{j+1}-x_{j} & =\left(\sum_{i=0} x_{i}\right)-1 \quad 2 \leq j \leq a-2
\end{aligned}
$$

and we can derive a lower bound of $F_{n}+F_{n+2}-a$.

4. $a=0$ or $a=1$. This is similar to case 2 except that the equations in (5) involving $y_{0}$ and $y_{1}$ are invalid. We remove the invalid equations from (5). Then if $b \geq 2$, we can work through (2) and (3) of Lemma 3.2 as we have done for case 2 and obtain a lower bound of $F_{n}+F_{n+2}+b-3$. If $b=0$ or 1 , then (5) reduces to (4) and we get the same lower bound of $F_{n}+F_{n+2}-2$.

In all cases, if $n>2$, we found a larger lower bound. Therefore we may take $F_{n}+F_{n+2}-n$ as a lower bound for all complete walks of $G_{n}$, for $n>2$. As we mentioned at the beginning of this section, this bound also holds for $n=2$.

We can say rather more.

Corollary 3.2.1 For $n>2, P$ is a minimal complete walk of $G_{n}$ of length $F_{n}+F_{n+2}-n$ if and only if $P$ starts in $V_{n}$, ends in $W$ and visits each vertex of $V_{n} \cup W$ exactly once. Furthermore one of the following two conditions holds:

1. $P$ starts in $V_{n}^{0}$ and ends in $V_{n}^{1}$.

2. $P$ starts in $V_{n}^{1}$ and ends in $V_{1}$.

Proof: Observe that the lower bounds we obtained for complete walks that either do not start in $V_{n}$ or do not end in $W$ are $>F_{n}+F_{n+2}-n$. Therefore from the proof of Lemma 3.2 we see that $P$ is a complete walk of length $F_{n}+F_{n+2}-n$ if and only if $P$ starts in $V_{n}$, ends in $W$ and visits each vertex of $V_{n}$ exactly once. So what remain to be shown are the two conditions on the start and end points of $P$.

Where could $P$ end? $P$ could not end in $V_{n}^{0}$ because otherwise vertices in $V_{0} \cup V_{1}$ are not visited by $P$. $P$ could not end in $V_{0}$ because then the only way to reach $V_{1}$ is from $V_{n}^{0}$. But $V_{0}$ is only reachable from $V_{n}^{0}$. Hence the single vertex in $V_{n}^{0}$ is visited more than once, contradicting our assumption about $P$.

Next we show that $P$ must start in $V=V_{n}^{0} \cup V_{n}^{1}$. To see this let us define $w_{1}, \ldots, w_{n-2}$ inductively as follows: $w_{1}=$ parent of the single vertex in $V_{n}^{0}, w_{j}=$ parent of $w_{j-1}$ that is not in $V_{n}$ for $2 \leq j \leq n-2$. We claim that if $P$ starts in $V_{n} \backslash V$, then all of $w_{j}(1 \leq j \leq n-2)$ are visited more than once. We prove this by induction. First, since $w_{1}$ has as its children the two vertices of $V$ and they are only reachable from $w$ by part 5 of Lemma 2.1, $w_{1}$ 
must be visited more than once. Now inductively assume $w_{j}$ is visited more than once for $1 \leq j<n-2$. Note that $w_{j}$ is one of the two children of $w_{j+1}$. As $w_{j}$ is visited more than once by the induction hypothesis, the total number of visits to the two children of $w_{j+1}$ is greater than 2. But the other parent $w$ of $w_{j}$ is in $V_{n}$ and thus is visited only once. So $w_{j+1}$ must be visited more than once. Thus by induction, our claim is proved. Now suppose $P$ ends in $V_{1}$. Observe that $w_{n-2}$ is the single vertex in $V_{2}$. Thus $w_{n-2}$ is reachable only from $V_{1}$ and $V_{n}^{1}$. Therefore since $P$ ends in $V_{1}, w_{n-2}$ is visited more than once implies that the single vertex of $V_{n}^{1}$ is visited more than once, a contradiction. Similarly if $P$ ends in $V_{n}^{1}$ we see that the single vertex of $V_{1}$ is visited more than once which again contradicts our assumption about $P$.

Lastly, we prove the connection between the start and the end points of $P$. Suppose $P$ starts in $V_{n}^{0}$. Then since $V_{0}$ and $V_{1}$ consist of the two children of $V_{n}^{0}$, we see that $P$ must end in $V_{n}^{1}$, because ending in $V_{1}$ would imply either $V_{n}^{0}$ is visited more than once or the only vertices visited by $P$ are those of $V_{n}^{0} \cup V_{0} \cup V_{1}$. In either case we arrive at a condition incompatible with our assumptions about $P$. Similarly, if $P$ starts in $V_{n}^{1}$ then $P$ must end in $V_{1}$.

\section{Algorithm}

We now give an algorithm that traces a complete walk in $G$ that satisfies the conditions of Corollary 3.2.1. It will then follow that our lower bound is achievable. Consequently the shortest word satisfying $\mathcal{P}_{f(n)}$ is of length $F_{n}+F_{n+2}$ for $n>1$. As in Section 3, we will assume $n>2$ throughout this section unless otherwise specified. The case $n=2$ is seen to be true by inspection.

We now introduce an order on the vertices of $G$ to facilitate descriptions of the algorithm and its proof. We think of $G$ as drawn in $n+1$ levels with $V_{0}$ at the top and $V_{n}$ at the bottom. Within each level, the vertices are ordered by their value as integers in binary. Large vertices are placed to the left. We view $G$ as a tree with root $V_{0}$ and "leaves" $V_{n}$ except that there are back edges from the "leaves" to the interior vertices. See Figure 1 for an example.

Now we can describe the algorithm as 


\section{Traverse $(\mathcal{T})$}

Input: $G_{n}$.

Output: A complete walk of $G_{n}$.

Begin

1. Start at $10 \ldots 0$ (the single vertex of $V_{n}^{0}$ ).

2. Go to $0 \ldots 0$ (the single vertex of $V_{0}$ ).

3. If current vertex has only one child (or equivalently current vertex ends in 1) then visit it.

else if the left child of the current vertex has not been visited then

visit the left child (left child is the one that ends in 1 ). else

visit the right child (right child is the one that ends in 0 ).

4. If the current vertex is $10 \ldots 01$ (the single vertex of $V_{n}^{1}$ ) then

Stop.

else

Repeat step 3.

End

An example walk traced out by the algorithm on $G_{5}$ in Figure 1 is as follows: $10000 \stackrel{0}{\rightarrow}$ $00000 \stackrel{1}{\rightarrow} 00001 \stackrel{0}{\rightarrow} 00010 \stackrel{1}{\rightarrow} 00101 \stackrel{0}{\rightarrow} 01010 \stackrel{1}{\rightarrow} 10101 \stackrel{0}{\rightarrow} 01010 \stackrel{0}{\rightarrow} 10100 \stackrel{1}{\rightarrow} 01001 \stackrel{0}{\rightarrow}$ $10010 \stackrel{0}{\rightarrow} 00100 \stackrel{0}{\rightarrow} 01000 \stackrel{1}{\rightarrow} 10001$ which gives the word 100000101010010001 .

We will henceforth refer to the algorithm just stated as algorithm $\mathcal{T}$. The following lemma gives the basic property of $\mathcal{T}$.

Lemma 4.1 The number of times $\mathcal{T}$ visits a vertex $v$ of a word graph $G=G_{n}$ is at most the number of children of $v$.

Proof: Suppose to the contrary, there exists $v$ that is visited more often than the number of its children and let us call such a vertex selfish. As $\mathcal{T}$ runs sequentially we can pick the first selfish vertex $v$. Could $v \in V_{0} \cup V_{1}$ ? If $v \in V_{0}$, then since the only edge to $v$ is from the start vertex and the start vertex cannot be visited more than once because it is the sibling of the vertex at which $\mathcal{T}$ terminates, we have that $v$ is unselfish, a contradiction. Similarly it is easy to see that $v \notin V_{1}$.

Now suppose $v \in V_{k}, 1<k \leq n$. There are two cases.

Case 1: $v$ ends in 1. In this case $v$ 's parent(s) has (have) two children and $v$ is the left child of its parent(s). By step 3 of $\mathcal{T}, v$ is never visited more than once. As $v$ has at least one child, $v$ cannot be selfish.

Case 2: $v$ ends in 0. Since $v$ ends in 0 and $k>1, v$ has two children. If $k=n$, then by Lemma 2.1 part 4, $v$ has one parent. So if $v$ is visited more than two times, so is $v$ 's parent. But the parent becomes selfish first. This contradicts that $v$ is the first selfish vertex. If $k<n$, then $v$ has two parents $v_{1} \in V_{n}$ and $v_{2} \notin V_{n}$. There are two cases here. By Lemma 2.1, both parents share the same children. 
Case 2a: If $v$ is the only child of both parents, then since $v$ is visited more than two times, one of the parents has been visited more than once and it becomes selfish before $v$. This is a contradiction.

Case 2b: If $v$ 's parents have two children, then by step 3 of $\mathcal{T} v$ must be the right child. Recalling that $v$ has 2 children, we have the total number of times $v$ and its left sibling were visited is $>3$. Since $v$ is the first selfish vertex, the parent $v_{2} \notin V_{n}$ can be visited at most two times, then $v_{1} \in V_{n}$ must have been visited at least two times. However $v_{1}$ has only one parent. So $v_{1}$ 's parent, say $w$, is visited at least twice. So $w$ must have two children. But then $v_{1}$ must be the right child of $w$ and $w$ must have been visited at least three times. Namely, $w$ becomes selfish before $v$ does. This is a contradiction.

Since $k$ is arbitrary, we conclude that such $v$ does not exist. So the lemma is proved.

The previous lemma implies the following property of $\mathcal{T}$.

Corollary 4.1 $\mathcal{T}$ visits each vertex of $V_{n}$ at most once.

Proof: Let us call a vertex $v \in V_{n}$ popular if $v$ is visited by $\mathcal{T}$ more than once. We will show that all vertices of $V_{n}$ are unpopular. Consider an arbitrary vertex $v \in V_{n}$. Let $w$ be a parent of $v$. Note that $v$ has only one parent by part 4 of Lemma 2.1. So $w$ is unique. If $v$ is the only child of $w$, then the unselfishness of $w$ implies the unpopularity of $v$. If $w$ has two children then there are two cases.

Case 1: $v$ ends in 1. Then $v$ has only one child. So $v$ is not popular since by Lemma 4.1 $v$ is not selfish.

Case 2: $v$ ends in 0. Then $v$ must be the right child of $w$. Since $w$ is unselfish, step 3 of $\mathcal{T}$ implies that $v$ is not popular. Thus the lemma is proved.

By Lemma 4.1, the two vertices in $V_{0} \cup V_{1}$ are unselfish. Since each of the two vertices has only one child, their unselfishness implies that they are visited by $\mathcal{T}$ at most once. Thus $\mathcal{T}$ visits each vertex in $W \cup V_{n}$ at most once. If we now prove that $\mathcal{T}$ indeed traces out a complete walk, then by Corollary 3.2.1, we conclude that it is a complete walk of length $F_{n}+F_{n+2}-n$ and the problem is solved. To do so we investigate how visitation of one vertex affects another vertex. It turns out that certain pairs of vertices are closely related in their visitation status. Such pair of vertices appears frequently in the sequel. Thus we define them as follows.

Definition 4.1 Suppose $v$ is not the start vertex (10..0), then we say that $w$ is the rightmost descendant of $v$ if $w$ satisfies the following three conditions.

1. $w \neq v$.

2. $w \in V_{n}$ and all edges on the shortest path from $v$ to $w$ have label 0.

3. $w$ is the only vertex in $V_{n}$ not equal to $v$ on the shortest path from $v$ to $w$. 
Two examples are shown in Figure 3. As usual we let the distance from a vertex $v$ to a vertex $w$ to be the length (number of edges) of the shortest path from $v$ to $w$. Observe that if $v^{\prime}$ is the rightmost descendant of $v$ and the distance from $v$ to $v^{\prime}$ is greater than 1 , then $v^{\prime}$ is also the rightmost descendant of the right child of $v$.
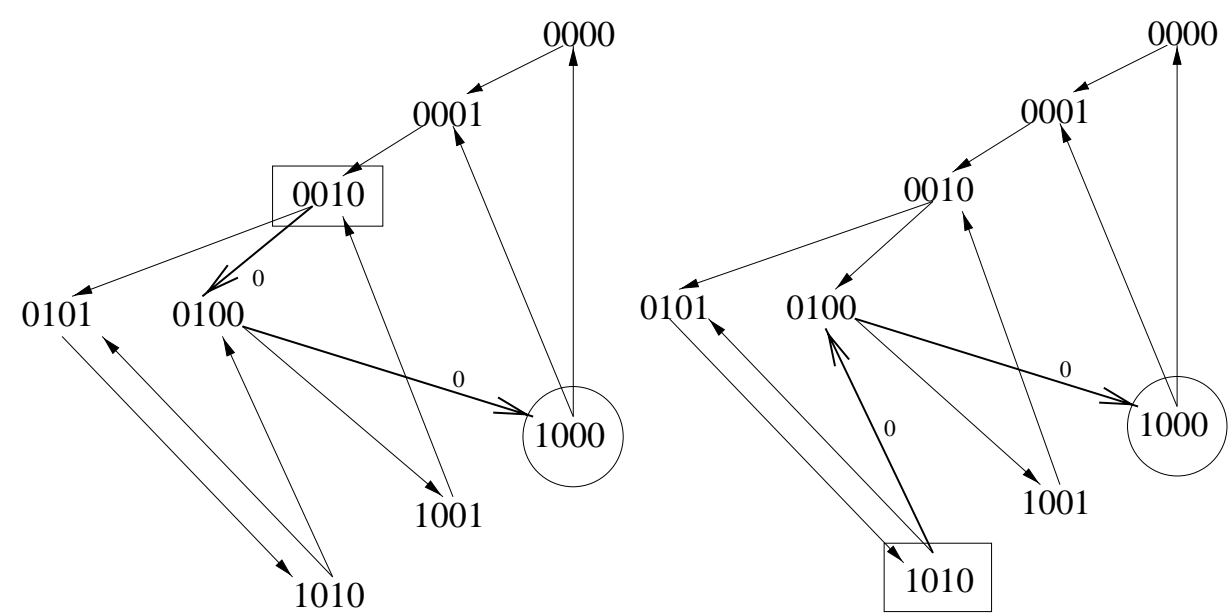

Figure 3: Circled vertices are the rightmost descendants of boxed vertices.

The basic relation between $v$ and its rightmost descendant is contained in the following lemma.

Lemma 4.2 Suppose vertex $v$ ends in 0 , and its rightmost descendant $v^{\prime}$ is not the start vertex. Then

1. If $v \in V_{n}$ is not visited by $\mathcal{T}$, then $v^{\prime}$ is not visited by $\mathcal{T}$.

2. If $v \notin V_{n}$ is visited by $\mathcal{T}$ only once, then $v^{\prime}$ is not visited by $\mathcal{T}$.

Proof: By step 1, step 2 and the first application of step 3 of $\mathcal{T}$, it is clear that all vertices in $V_{0} \cup V_{1}$ are visited by $\mathcal{T}$. Therefore we assume $v \notin V_{0} \cup V_{1}$ in the rest of the proof. We proceed by induction on the distance $d$ from $v$ to $v^{\prime}$.

$d=1$ : In this case we have $v \notin V_{n}$ and $v$ has two children. Since $v^{\prime}$ is not the start vertex and is the right child of $v$, then by step 3 of $\mathcal{T}, v^{\prime}$ is not visited because $v$ is visited only once.

$d=k>1$ : Since $v$ ends in $0, v \notin V_{0} \cup V_{1}, v$ has two children. Since $k>1$, the right child $w$ of $v$ is not in $V_{n}$. Let $u$ be the other parent of $w$. If $v$ is in $V_{n}$ and is not visited by $\mathcal{T}$, then Lemma 4.1 implies that $w$ is visited only once because $w$ is $u$ 's right child and $u$ is unselfish. Suppose $v \notin V_{n}$ and is visited by $\mathcal{T}$ only once. Then the other parent $u$ of $w$ is in $V_{n}$ and Corollary 4.1 implies that $w$ is visited only once due to the unpopularity of $u$. Since the distance from $w$ to $v^{\prime}$ is less than $d$, by the induction hypothesis $v^{\prime}$ is not visited. This proves the lemma.

Next we need 
Lemma 4.3 Suppose the rightmost descendant $v^{\prime}$ of $v$ is the start vertex. Then $v$ is visited by $\mathcal{T}$ at most once.

Proof: If $v \in V_{n}$, then Corollary 4.1 implies this lemma. So suppose $v \notin V_{n}$. We prove this case by induction on the distance from $v$ to $v^{\prime}$. It is easy to see that the result holds for vertices of $V_{0} \cup V_{1} \cup V_{2}$ since the only vertices in $V_{n}$ that contain an edge to them are the start and end vertex. Therefore we inductively assume the lemma is true for distance $d>n-3$. For distance $d \leq n-3, v$ must be the right child of its parents because the start vertex is the rightmost vertex of $V_{n}$ and $d \leq n-3$. Since $v$ has at most 2 parents, if $v$ is visited more than once, one of its parents must be visited more than once because $v$ is the right child of both parents. But this contradicts the induction hypothesis. So $v$ is visited at most once. This proves the lemma.

Lemma 4.3 can be sharpened to

Lemma 4.4 Suppose the rightmost descendant $v^{\prime}$ of $v$ is the start vertex, then $v$ is visited by $\mathcal{T}$ exactly once.

Proof: The cases where $v \in V_{0} \cup V_{1}$ are trivially true. We prove the other cases by induction on the distance $d$ from $v$ to $v^{\prime}$.

$d=1$ : In this case $v$ is the parent of the end vertex. Since there are only finitely many vertices in $G_{n}$, by Lemma $4.1, \mathcal{T}$ terminates. Therefore $v$ is visited exactly once.

$d>1$ : There are two cases here.

Case 1: $v \notin V_{n}$. Suppose $v$ has not been visited. Then Corollary 4.1 and step 3 of $\mathcal{T}$ implies that the right child of $v$ is not visited. This contradicts the induction hypothesis. So by Lemma 4.3, $v$ is visited exactly once.

Case 2: $v \in V_{n}$. If $v$ is not visited, then by case 1 above, the right child of $v$ is not visited. This again contradicts the induction hypothesis. So $v$ is visited exactly once. By induction, the lemma is proved.

Putting previous results together, we can now prove the main theorem.

Theorem $4.1 \mathcal{T}$ traces out a minimal complete walk in $G_{n}$ of length $F_{n}+F_{n+2}-n$.

Proof: We first prove that all vertices of $V_{n}$ are visited. Assume to the contrary that some vertices of $V_{n}$ are not visited. Pick the rightmost such vertex $v$. Note that $v \notin V_{n}^{0} \cup V_{n}^{1}$ as these are the start and end points of $\mathcal{T}$. For vertices $v \in V_{n} \backslash\left(V_{n}^{0} \cup V_{n}^{1}\right)$ there are two cases.

Case 1: $v$ ends in 1. Then $v$ 's parent has two children and $v$ is the left child. By step 3 of $\mathcal{T}$, if $v$ is not visited, then $v$ 's right sibling cannot have been visited. This is impossible as $v$ is supposed to be the rightmost unvisited vertex.

Case 2: $v$ ends in 0 . Then let $v^{\prime}$ be the rightmost descendant of $v$. If $v^{\prime}$ is not the start vertex, then by Lemma $4.2 v^{\prime}$ is not visited. Note that $v^{\prime}$ is obtained from $v$ by removing a positive number of symbols from the left end of $v$ and appending the same number of zeros to the right end of $v$. Therefore by repeated applications of Lemma 4.2, we get a sequence of 
vertices $v^{\prime}, v^{\prime \prime}, \ldots$ such that eventually we arrive at an unvisited vertex $v^{(m)}$ whose rightmost descendant $v^{(m+1)}$ is the start vertex. However Lemma 4.4 now implies that $v^{(m)}$ is visited by $\mathcal{T}$, a contradiction. This proves that $\mathcal{T}$ visits all vertices of $V_{n}$.

Now we assume inductively that $\mathcal{T}$ visits all vertices of $V_{k}, 2<k \leq n$. Suppose some vertices of $V_{k-1}$ are not visited. Pick the rightmost such vertex. There are two cases.

Case 1: $v$ ends in 1. The same argument as in case 1 above shows this case is impossible.

Case 2: $v$ ends in 0. Since $v \notin V_{0} \cup V_{1}, v$ has two children. Then Corollary 4.1 implies that the right child of $v$ is not visited. This contradicts the inductive hypothesis. So this case is also impossible.

Thus $\mathcal{T}$ visits all vertices of $V_{k-1}$. By induction, $\mathcal{T}$ visits all vertices of $V_{i}, 2 \leq i \leq n$. By step 1 and step 2 of $\mathcal{T}$, it also visits the vertices of $V_{0}$ and $V_{1}$. Further by Lemma 4.1, each vertex in $V_{0} \cup V_{1}$ is visited exactly once. So it traces a complete walk in $G_{n}$ which satisfies the condition of Corollary 3.2.1. Therefore we conclude that the path it traced is a minimal complete walk of length $F_{n}+F_{n+2}-n$.

A table of words produced by the algorithm for $1 \leq n \leq 7$ is given below. The cases $n=1$ and $n=2$ are produced by hand.

\begin{tabular}{|l|l|}
\hline 1 & 10 \\
\hline 2 & 1001 \\
\hline 3 & 1000101 \\
\hline 4 & 10000101001 \\
\hline 5 & 100000101010010001 \\
\hline 6 & 10000001010100101000100100001 \\
\hline 7 & 10000000101010100101000101000010010010001000001 \\
\hline
\end{tabular}

\section{$5 \quad$ Remarks on generalizations}

It would be interesting to see if some of the ideas presented here could be used to obtain more general results on feasible functions. In particular lower bounds on the length of minimal words for $f$ satisfying a linear recurrence. It seems that the idea of partitioning the vertices of the word graph into "levels" may be useful in this context.

\section{Acknowledgement}

Theorem 4.1 proved a conjecture on length which originated from David Swart's computation of the minimal words of order $n$ for $1 \leq n \leq 6$.

\section{References}

[A94] Allouche, J.-P. Sur la complexité des suites infinies, Bull. Belg. Math. Soc. 1 (1994), 133-143. 
[B46] de Bruijn, N. G. A combinatorial problem, Nederl. Akad. Wetensch. Proc. 49 (1946), $758-764$.

[B75] de Bruijn, N. G. Acknowledgement of priority to C. Flye Sainte-Marie on the counting of circular arrangements of $2 n$ zeros and ones that show each $n$-letter word exactly once, TH-Report 750WSK-06, Eindhoven University of Technology the Netherlands (1975), 1-14.

[G46] Good, I. J. Normally recurring decimals, J. London Math. Soc. 21 (1946), 167-169.

[F82] Fredricksen, H. A survey of full length nonlinear shift register cycle algorithms, SIAM Rev. 24 (1982), 195-221.

[F94] Flye-Sainte Marie, C. Solution to problem number 58, L'Intermédiaire des Mathématiciens 1 (1894), 107-110.

[R83] Rauzy, G. Suite à termes dans un alphabet fini, Séminaire de Théorie des Nombres de Bordeaux (1982-1983), nº 25. 